5. Stephans C, Jung $\mathrm{K}$, Diamandis $\mathrm{PE}$, Rithtenhouse $\mathrm{HG}$, Lein $\mathrm{M}$, Loening AS. Prostate specific antigen, its molecular forms, and other kallikrein markers for detection of prostate cancer. Urology 2002; 59: 2-8.

6. Catalona WJ, Smith DS, Ornstein DK. Prostate cancer detection in men with serum PSA concentrations of 2.6 to $4.0 \mathrm{ng} / \mathrm{ml}$ and benign prostate examination. Enhancement of specificity with free PSA measurements. JAMA 1997; 277: 1452-6.

7. Catalona WJ, Partin AW, Finlay JA, Chan DW, Rittenhouse HG, Wolfert RL, Woodrum DL. Use of percentage of free prostaticspecific antigen to identify men at high risk of prostate cancer when PSA levels are 2.51 to $4.0 \mathrm{ng} / \mathrm{ml}$ and digital rectal examination is not suspicious of cancer: an alternative model. Urology 1999; 220-4.

8. Littrup PJ, Kane RA, Metlin CJ, Murphy GP, Lee F, Toi A, Badalament R, Babaian RJ. Cost-effective prostate cancer detection: reduction of low yield biopsies. Cancer 1994 74: 3146-58.

9. Okihara K, Fritsche HA, Ayala A, Johnston DA, Allard WJ, Babaian RJ. Can complexed prostate specific antigen and prostatic volume enhance prostate cancer detection in men with total prostate specific antigen between 2.5 and 4.0ng/ml. J Urol 2001; 165 : 193-6.

10. Haese A, Graefen M, Noldus J, Hammerer P, Huland E, Huland H. Prostatic volume and ratio of free-to-total prostate specific antigen in patients with prostatic cancer or benign prostatic hyperplasia. J Urol 1997; 2189-92.

11. Gann PJ, Hennekens CH, Stampfer MJ. A prospective evaluation of plasma prostate specific antigen for detection of prostatic cancer. JAMA 1995; 273: 289-94.

12. Martins ACP, Bovo TJB, Reis RB, Paschoalin EL, Cologna Aj, Suaid HJ. Performance of PSA and of PSA density in the diagnosis of prostate carcinoma. Acta Cir Bras 2002; 17(S): 7-11.

13. Stephans C, Lein M, Jung K, Rudolph B, Schnorr D, Loening SA. The influence of prostate volume on the ratio of free to total prostate specific antigen in serum of patients with prostate carcinoma and benign prostate hyperplasia. Cancer 1997; 79: 104-9.

14. Oesterling JE, Jacobsen SJ, Chute CG, Guess HA, Girman CJ, Panser LA, Lieber MM. Serum PSA in a community-based population of healthy men: establishment of agespecific reference range. JAMA 1993; 270: 860-4.

15. Richardson TD, Oesterling JE. Age-specific reference ranges for serum prostate-specific antigen. Urol Clin North Am 1997;24:339-51.

16. Cheli CD, Levine R, Cambetas DR, Kolker JD, Roberts SB. Age-related reference ranges for complexed prostate-specific antigen and complexed/total prostate-specific ratio: results from East Texas Medical Center Cancer Institute screening campaign.

Urology 2002; 60 (Suppl A): 53-9. Martins ACP, Rodríguez J AA, Reis RB, Bovo TB, Suaid HJ, Cologna AJ, Paschoalin EL. PSA livre e volume prostático no diagnóstico do cancer da próstata. Acta Cir Bras [serial online] 2003 vol 18 suppl 5. Disponível em www.scielo.br/acb

RESUMO - Objetivo: Investigar a influencia do volume prostático no desempenho do PSA total (tPSA) e livre (fPSA) no diagnóstico do adenocarcinoma prostático. Métodos: 188 patients foram submetidos à biópsias prostáticas guiadas por ultra-som trans-retal (10-12 fragmentos) por apresentarem toque retal alterado e/ou tPSA entre 2,5 e 10ng/ml. A idade média foi $65,7 \pm 8,7$ anos. A prevalência do câncer foi de $19 \%$ (19/100) em pacientes com próstatas $>40 \mathrm{ml}$ (GI) e 29,5\% (26/88) naqueles com próstatas <40ml (GII). Analisamos a sensibilidade e a especificidade do tPSA em corte de 2,5ng/ml e $4 \mathrm{ng} / \mathrm{ml}$ bem como a influência do fPSA nos dois grupos de pacientes. Resultados: No grupo GI a sensibilidade e a especificidade do tPSA foram de $94,4 \%$ e $19,5 \%$ no corte de $4 \mathrm{ng} / \mathrm{ml}$ e $100 \%$ e $6 \%$ no corte de 2,5ng/ml. Para o grupo GII os valores correspondentes foram $76,5 \%$ e $62,9 \%$, e $100 \%$ e $19,3 \%$. No GI a aplicação da fração f/tPSA, corte de $19 \%$, manteve a sensibilidade do teste acima de $90 \%$ e elevou a especificidade para 46,2\% no corte de $4 \mathrm{ng} / \mathrm{ml}$ e $32,9 \%$ no corte de $2,5 \mathrm{ng} / \mathrm{ml}$. No GII a fração f/tPSA não foi capaz de elevar a especificidade do tPSA sem afetar significativamente a sensibilidade. Porém, neste grupo o uso do quociente f/tPSA de $16 \%$ elevou a especificidade do tPSA, corte de $2,5 \mathrm{ng} / \mathrm{ml}$, para $46,7 \%$ para sensibilidade acima de $90 \%$. Conclusão: Recomenda-se estratificar os pacientes segundo o volume prostático para definir o corte do tPSA. O tPSA no corte de $2,5 \mathrm{ng} / \mathrm{ml}$, associado ao f/TPSA de $19 \%$ em próstatas $>40 \mathrm{ml}$ e de $16 \%$ para próstatas $<40 \mathrm{ml}$ representou melhor opção para indicação de biópsia que o tPSA no corte de $4 \mathrm{ng} / \mathrm{ml}$ associado ou não à fração f/tPSA. DESCRITORES: Antígeno prostático específico. PSA, rastreamento. Câncer, próstata. Adenocarcinoma.

Correspondence address:

Antonio Carlos Pereira Martins

Hospital das Clínicas - Departamento de Cirurgia

Av. Bandeirantes, 3.900, $9^{\circ}$ Andar

Ribeirão Preto, SP, CEP: 14048-900

9-ARTIGOORIGINAL

\title{
Efeito do cloridrato de oxibutinina na hiperatividade vesical conseqüente a cistite hemorrágica ${ }^{1}$
}

\author{
Mizuma, EK ${ }^{2}$; Takeshita, MS $^{3}$; Haylton Jorge Suaid ${ }^{4}$ Antonio Carlos Pereira Martins \\ Silvio Tucci $\mathrm{Jr}^{6}$ Adaulto José Cologna ${ }^{6}$ Gonçalves, MA ${ }^{7}$.
}

\footnotetext{
Mizuma EK, Takeshita MS; Suaid HJ, Martins ACP, Tucci Jr S, Cologna AJ, Gonçalves MA. Efeito do cloridrato de oxibutinina na hiperatividade vesical conseqüente a cistite hemorrágica. Acta Cir Bras [serial online] 2003 vol 18 suppl 5. Disponível em www.scielo.br/acb.

RESUMO - Introdução: A oxibutinina atua como agente anticolinérgico que tem ação anti-muscarínica e, principalmente, ação antiespasmódica na musculatura lisa vesical. Assim, ela causa aumento da capacidade vesical e diminui a frequência miccional e bloqueia o estímulo inicial da micção. Objetivo: Verificar se a oxibutinina atua sobre a hiperatividade vesical causada pela cistite hemorrágica, dependente do óxido nítrico. Métodos: Foram estudados dois grupos de animais. O controle com 5 ratas e o experimental com 10 ratas, cujos pesos variaram entre $200 \mathrm{~g}$ a $250 \mathrm{~g}$. A cistite hemorrágica foi provocada pela injeção intraperitoneal de ciclofosfamida $200 \mathrm{mg} /$
} 
$\mathrm{kg}$, na véspera do experimento. Após 24 horas, as ratas foram anestesiadas com uretana 1,25mg/kg. A seguir, foi feita cistostomia com cateter P-50. Esse cateter foi conectado em Y a uma bomba de infusão contínua com fluxo de água de $0,3 \mathrm{ml} / \mathrm{min}$ e a um polígrafo para o registro da cistometria. O registro cistométrico foi feito com a velocidade do papel de $0,05 \mathrm{~cm} / \mathrm{seg}$, com sensibilidade de 20 e calibração para um curso de $60 \mathrm{~mm}$ para uma pressão de $100 \mathrm{mmHg}$. Os parâmetros estudados foram: freqüência de contração (Fc), intensidade das contrações (Ic), tempo de enchimento vesical (Te), tempo de contração vesical (Tc) e capacidade vesical (Cv), que foi determinado pelo Te x Fluxo. Esses parâmetros foram determinados por suas médias durante o período de observação de 10 min. Após o registro, foi infundido por gavagem $71 \mathrm{mg} / \mathrm{kg}$ de cloridato de oxibutinina. Uma hora depois foi feita nova cistometria. A análise estatística foi feita pelo método de Kruskal-Wallis que comparou os valores do grupo controle com o experimental. O p foi considerado significante quando menor que 0,05. Resultados: A comparação entre os dois grupos dos parâmetros estudados antes da infusão do cloridrato de oxibutinina mostrou: $\mathrm{Fc}-\mathbf{p = 0 , 0 0 7}$; $\mathrm{Ic}-\mathbf{p = 0 , 0 0 0 2}$; Te $-\mathbf{p = 0 , 7 6 8}$; $\mathrm{c}-\mathbf{p = 0 , 4 9 2 ;} \mathrm{Cv}-\mathbf{p = 0 , 0 5 6}$ A comparação dos parâmetros estudados depois da droga mostrou: $\mathrm{Fc}-\mathbf{p = 0 , 0 5 5}$; Ic $-\mathbf{p = 0 , 0 0 0 2}$; Te $-\mathbf{p = 0 , 9 5 7}$; $\mathrm{T}$ - $\mathbf{p = 0 , 1 8 1 ;} \mathrm{Cv}-\mathbf{p = 0 , 2 0 6}$. Conclusões: O cloridrato de oxibutinina nesse modelo experimental atuou de forma a alterar somente a frequiência das miccções, controlando a hiperatividade e não promovendo alterações nos demais parâmetros estudados.

DESCRITORES: Cistite. Polaquiúria. Oxibutinina. Ciclofosfamida

\section{INTRODUÇÃO}

Os antagonistas muscarínicos são considerados agentes parassimpatolíticos por reduzirem ou abolirem seletivamente os efeitos da estimulação parassimpática. A forma de ação dos antagonistas é por competição com a acetilcolina nos receptores muscarínicos. O Cloridrato de Oxibutinina atua como agente anticolinérgico e é uma amina terciária que tem ação anti-muscarínica e, principalmente, ação antiespasmódica na musculatura lisa vesical. Não se sabe especificamente em qual receptor muscarínico ela age. Entretanto, acredita-se que tenha maior afinidade pelos receptores M3 ${ }^{1}$. No músculo detrusor os receptores parassimpáticos presentes são do tipo M2-M3, embora a mioria sejja $\mathrm{M} 2^{1,2}$. Assim, o cloridrato de oxibutinina atua de forma a aumentar a capacidade vesical, diminuir a frequência miccional e atrasar o estímulo inicial da micção. A indicação de uso é para os pacientes com hiperratividade vesical de origem motora ou sensorial, como também na hiperatividade vesical hiperreflexa, cujo objetivo é promover o bloqueio do detrusor e consequentemente tratar a urgência miccional, bem como a incontinência urinária de urgência miccional ${ }^{3}$.

\section{OBJETIVO}

Verificar a atuação do cloridrato de oxibutinina sobre a hiperatividade vesical causada pela cistite hemorrágica dependente do óxido nítrico.

\section{MÉTODOS}

\section{1) ANIMAIS}

Foram utilizadas 16 ratas Wistar com peso aproximado de $200 \mathrm{~g}$, que foram divididas em 2 grupos.

- Grupo I (GI controle): constituído por 6 ratas normais, que receberam $2,5 \mathrm{ml}$ de $\mathrm{NaCl}$ a $0,9 \%$ (mesmo volume de ciclofosfamida administrada no grupo experimental) por via intra-peritoneal. Depois da cistometria controle, injetou-se, via orogástrica, aproximadamente, $1,0 \mathrm{ml}$ de solução salina a $0,9 \%$ (mesmo volume do cloridrato de oxibutinina).
- Grupo II (GII): 10 ratas receberam o cloridrato de oxibutinina na concentração de $71 \mathrm{microgr} / \mathrm{kg}$ diluídos em $1,0 \mathrm{ml}$ de solução fisiológica através de sonda orogástrica.

\section{2) INDUÇÃO DA CISTITE HEMORRÁGICA}

Ratas normais receberam uma injeção intraperitoneal de ciclofosfamida numa dose de $200 \mathrm{mg} /$ $\mathrm{kg}$ diluída em soro fisiológico na concentração de $20 \mathrm{mg} / \mathrm{ml}$ que foi dada $24 \mathrm{~h}$ antes da determinação da função vesical ${ }^{4}$.

\section{3) ANESTESIA}

As ratas foram anestesiadas com uretana na dosagem de $1,250 \mathrm{mg}$ por kilo de peso, via intraperitonial.

\section{4) CIRURGIA}

Cistostomia: Inicialmente foi feita tricotomia abdominal. Depois a laparotomia com exposição, reparo e abertura do fundo vesical, local onde se introduziu e fixou um cateter de polietileno PE50. Esse cateter foi exteriorizado por contra abertura na região dorso lateral direita onde também foi fixado. Serviu para se fazer as medidas das pressões vesicais.

\section{5) REGISTRO DAS PRESSÕES VESICAIS}

O sistema para se fazer o registro das pressões veisicais foi composto por uma bomba de infusão continua regulada para um fluxo de $0,3 \mathrm{ml} /$ minuto, conectada em $\mathrm{Y}$ ao cateter de cistostomia e a um polígrafo Narco-Biosystem. Determinou-se assim, o comportamento vesical durante 10 minutos, registrando-se o ciclo miccional de enchimento e esvaziamento vesical ${ }^{5}$. Os registros foram feitos após a cistostomia e uma hora após a infusão gátrica do cloridrato de oxibutinina.

\section{6 ) ANÁLISE ESTATÍSTICA}

Os resultados dos parâmetros estudados nos registros miccionais das 16 ratas nos dois períodos estudados foram analisados e comparados através do método de Wilcoxon dentro do mesmo grupo e de Kruskal-Wallis entre os dois grupos. Considerou-se significante a relação para $\mathrm{p}<0,05$.

\section{RESULTADOS}

1. Avaliação estatística (Wilcoxon) das cistometrias de cada grupo

TABELA 1. Parâmetros urodinâmicos observados no grupo GI (controle).

\begin{tabular}{c|c|c|c|c|c}
\hline & \multicolumn{2}{|c|}{ Antes } & \multicolumn{2}{c|}{ Depois } & \\
\hline Parâmetros & Média & $\begin{array}{c}\text { Desvio } \\
\text { Padrão }\end{array}$ & Média & $\begin{array}{c}\text { Desvio } \\
\text { Padrão }\end{array}$ & p \\
\hline CV $\left(\mathrm{mm}^{3}\right)$ & 321,1 & 144,2 & 348,1 & 151,0 & 0,5 \\
\hline FC $($ cont $/ 10 \mathrm{~min})$ & 7,5 & 3,2 & 7,3 & 2,6 & 0,8 \\
\hline $\mathbf{I C}\left(\mathrm{mmH}_{2} \mathrm{O}\right)$ & 316,1 & 41,6 & 271,9 & 39,4 & 0,06 \\
\hline TC $(\mathrm{seg})$ & 18,6 & 5,8 & 22 & 9,3 & 0,1 \\
\hline TE $(\mathrm{seg})$ & 63,9 & 28,2 & 69,6 & 30,2 & 0,3 \\
\hline
\end{tabular}

TE: Tempo de Enchimento, CV: Capacidade Vesical, IC: Intensidade da Contração, TC: Tempo de contração, FC: Frequiência de Contrações

1. Trabalho realizado no setor de Cirurgia Experimental do Departamento de Cirurgia e Anatomia - HCFMRP-USP

2. Aluno de Iniciação Científica do Depto de Cirurgia e Anatomia da FMRP-USP - CAPES

3. Aluno de Graduação do Curso de Medicina da FMRP-USP

4. Prof. Associado do Depto de Cirurgia e Anatomia - Disciplina de Urologia da FMRP - USP

5. Prof. Titular do Depto de Cirurgia e Anatomia - Disciplina de Urologia da FMRP - USP

6. Prof. Doutor do Depto de cirurgia e Anatomia - Disciplina de Urologia da FMRP - USP

7. Pós-Graduando do Depto de Cirurgia e Anatomia da FMRP - USP 
TABELA 2. Parâmetros observados nas cistometrias de ratas com cistite hemorrágica antes e após a infusão gástrica do cloridrato de oxibutinina (GII).

\begin{tabular}{c|c|c|c|c|c}
\hline & \multicolumn{2}{|c|}{ Antes } & \multicolumn{2}{c|}{ Depois } & \\
\hline Parâmetros & Média & $\begin{array}{c}\text { Desvio } \\
\text { Padrão }\end{array}$ & Média & $\begin{array}{c}\text { Desvio } \\
\text { Padrão }\end{array}$ & p \\
\hline CV $\left(\mathrm{mm}^{3}\right)$ & 128 & 92,3 & 191 & 109,2 & 0,0039 \\
\hline FC $($ cont $/ 10 \mathrm{~min})$ & 15,8 & 6,6 & 12,8 & 5,5 & 0,0273 \\
\hline $\mathbf{I C}\left(\mathrm{mmH}_{2} \mathrm{O}\right)$ & 33,0 & 13 & 38,7 & 19,7 & 0,4316 \\
\hline $\mathbf{T C}(\mathrm{seg})$ & 17,5 & 8 & 18,0 & 13,3 & $>0,999$ \\
\hline $\mathbf{T E}(\mathrm{seg})$ & 25,6 & 18,4 & 38,1 & 21,9 & 0,0039 \\
\hline
\end{tabular}

2. Análise estatística comparando os 2 grupos (Kruskal-Wallis).

TABELA 3. Análise estatística dos parâmetros estudados entre os Grupos GI e GII antes da administração da droga.

\begin{tabular}{|c|c|c|c|c|c|}
\hline \multirow[b]{3}{*}{ Parâmetros } & \multicolumn{4}{|c|}{ GRUPOS } & \multirow[b]{3}{*}{$\mathbf{p}$} \\
\hline & \multicolumn{2}{|c|}{ GI } & \multicolumn{2}{|c|}{ GII } & \\
\hline & MÉDIA & $\mathbf{S}$ & MÉDIA & $\mathbf{S}$ & \\
\hline $\mathbf{C V}\left(\mathrm{mm}^{3}\right)$ & 321,1 & $\pm 144,2$ & 128 & $\pm 92,3$ & 0,056 \\
\hline $\mathbf{F C}($ cont $/ 10 \mathrm{~min})$ & 7,5 & $\pm 3,2$ & 15,8 & $\pm 6,6$ & 0,007 \\
\hline IC $(\mathrm{mmH} 2 \mathrm{O})$ & 316 , & $\pm 41,6$ & 33 & \pm 13 & 0,0002 \\
\hline TC (seg) & 18,6 & $\pm 5,8$ & 17,5 & \pm 8 & 0,492 \\
\hline TE (seg) & 63,9 & $\pm 28,2$ & 25,6 & $\pm 18,4$ & 0,0768 \\
\hline
\end{tabular}

S=desvio padrão

TABELA 4. Análise estatística dos parâmetros estudados entre os grupos GI e GII após a administração da droga.

\begin{tabular}{|c|c|c|c|c|c|}
\hline \multirow[b]{3}{*}{ Parâmetros } & \multicolumn{4}{|c|}{ GRUPOS } & \multirow[b]{3}{*}{$\mathbf{p}$} \\
\hline & \multicolumn{2}{|c|}{ GI } & \multicolumn{2}{|c|}{ GII } & \\
\hline & MÉDIA & $\mathbf{S}$ & MÉDIA & $\mathbf{S}$ & \\
\hline $\mathbf{C V}\left(\mathrm{mm}^{3}\right)$ & 348,1 & \pm 151 & 191 & $\pm 109,2$ & 0,206 \\
\hline $\mathbf{F C}(\operatorname{cont} / 10 \mathrm{~min})$ & 7,3 & $\pm 2,6$ & 12,8 & $\pm 5,5$ & 0,055 \\
\hline IC $(\mathrm{mmH} 2 \mathrm{O})$ & 271,9 & $\pm 39,4$ & 38,7 & $\pm 19,7$ & 0,0002 \\
\hline TC (seg) & 22,0 & $\pm 9,3$ & 18 & $\pm 13,3$ & 0,181 \\
\hline TE (seg) & 69,6 & $\pm 30,2$ & 38,1 & $\pm 21,9$ & 0,768 \\
\hline
\end{tabular}

$\mathrm{S}=$ desvio padrão

\section{DISCUSSÃO}

Na tabela 1 estão agrupadas as médias dos valores dos parâmetros estudados nas cistometrias de 6 ratas do grupo controle, bem como o desvio padrão, o valor de "p" e o nível de significância do teste de Wilcoxon, que não identificou diferenças estatísticas na comparação dos resultados antes e após a infusão gástrica de solução salina a $0,9 \%$.
A avaliação cistométrica do grupo experimental (GII), com uso do cloridrato de oxibutinina, em 10 ratas com cistite hemorrágica estão agrupadas na tabela 2. Observa-se que o teste de Wilcoxon identificou diferenças muito significantes em 2 parâmetros, significantes em 1, e sem alterações significantes em 2 parâmetros.

A cistite provocada pela injeção de ciclofosfamida alterou no grupo experimental a $\mathrm{Fc}$ e Ic, não alterando os demais parâmetros quando comparamos com o Grupo Controle (tabela 3). Portanto, ao analisar o resultado após a administração do cloridrato de oxibutinina (tabela 4), percebemos que a droga atuou de forma a alterar a frequiência de micções, não promovendo alterações nos demais parâmetros estudados.

\section{CONCLUSÕES}

O cloridrato de oxibutinina atuou de forma a diminuir a hiperatividade vesical, aumentar a capacidade vesical e aumentar o tempo de enchimento vesical.

Houve diminuição acentuada da força de contração do músculo detrusor em relação ao controle normal

\section{REFERÊNCIAS}

1. Yamanishi T; Chapple CR; Chess-Willians R: With muscarinic receptor is important in the bladder? World J Urol 2001; 19(5):299-306.

2. Kondo S, Morita T: A study of muscarinic cholinergic receptor subtypes içn human detrusor muscle using radioligand binding techiniques. Nippon Hinyokika Gakkai Zasshi 1993 Jul; 84(7): 1255-61.

3. O'Leary,M; Erickson, JR;Smith, CP; McDermott, C; Horton, J; Chancellor, MB. Effect of controlled-release oxybutynin in neurogenic bladder function in spinal cord injury. J Spinal Cord Med 2003; 26(2): 159-62.

4. Souza-Filho, MB; Lima, MV; Pompeu, MM; Balljo, G; Cunha, FQ; Ribeiro, RA. Involvemente of nitric oxide in the pathogenesis of cyclophosphamide-cuced hemorragic cystitis. Am J Pathol 1997; 150: 1247-56.

5. Suaid, HJ; Martins, ACP; Nina-Rocha, J; Cologna, AJ; Tucci Jr, S. Comparação da função uretral após cistectomia simples em ratas e cistoprostatectomia em ratos. Acta Cir Bras 1998; 13: 25.

Mizuma EK, Takeshita MS; Suaid HJ, Martins ACP, Tucci Jr S, Cologna AJ, Gonçalves MA. Effects of oxybutynin in bladder hyperactivity caused by hemorrhagic cystitis. Acta Cir Bras [serial online] 2003 vol 18 suppl 4. Available in www.scielo.br/acb.

ABSTRACT - Background: The oxybutynin is an anticholinergic agent that binds to muscarinic receptors of bladder muscle promoting an antispasmodic effect. As a consequence there is an increase in bladder capacity, reduction in frequency and a blockade in the initial stimuli of miction. Objective: To verify the action of oxybutynin on bladder overactivity due to hemorrhagic cystitis. Methods: Hemorrhagic cystitis was provoked through an intraperitoneal injection of $200 \mathrm{mg} / \mathrm{kg}$ of cyclophosphamide in 10 female rats, weighting $200-250 \mathrm{~g}, 24 \mathrm{~h}$ before the experiment. The control group of 5 female rats received an intraperitoneal injection of $0.5 \mathrm{ml}$ of saline. In the experiment the animals were anesthetized with $1.25 \mathrm{mg} / \mathrm{kg}$ of urethane followed by a cystostomy with a P-50 catheter connected in $\mathrm{Y}$ to an infusion pump (rate of infusion $-0.3 \mathrm{ml} / \mathrm{min}$ ) and to a polygraph to register bladder pressure. The parameters studied were: frequency of bladder contraction $(\mathrm{Fc})$, contraction intensity $(\mathrm{Ci})$, time of bladder filling (Tf) and contraction $(\mathrm{Tc})$, and vesical capacity $(\mathrm{Vc})$ ( $\mathrm{Tf} \times \mathrm{x}$ flow). We determined the mean value for each parameter following of $10 \mathrm{~min}$ of observation. After these determinations all rats received $71 \mathrm{mg} / \mathrm{kg}$ of oxybutynin chloride through nasogastric tubing and $1 \mathrm{~h}$ afterward the measures were repeated. The data were compared with the Kruskal-Wallis test considering significant a $\mathrm{p}<0.05$. Results: A comparison both 
groups (control versus experimental) before the use of oxybutynin showed the following values of $\mathrm{p}$ : $\mathrm{Fc}-0.007 ; \mathrm{Ci}-0.0002$; Tf 0.768 ; Tc $-0.492 ; \mathrm{Vc}-0.055$. After the use of oxybutynin the corresponding values were: $\mathrm{Fc}-\mathrm{p}=0.055 ; \mathrm{Ci}-\mathrm{p}=0.0002 ; \mathrm{Tf}-0.957$; $\mathrm{Tc}-\mathrm{p}=0.181 ; \mathrm{V} c-\mathrm{p}=0.206$. Conclusion: The oxybutynin chloride was able to control bladder overactivity expressed by a significant reduction in frequency, but no changes in other parameters.

KEY WORDS: Cystitis, frequency. Oxybutynin. Cyclophosphamide.

\title{
CORRESPONDÊNCIA:
}

Haylton Jorge Suaid

Hospital das Clínicas - Departamento de Cirurgia

Av. Bandeirantes, $3900-9^{\circ}$ Andar

Ribeirão Preto, CEP: 14048-900

10 - ARTIGO ORIGINAL

\section{Ação do citrato de sildenafil sobre a função uretral de ratas com desnervação vesical ${ }^{1}$}

\author{
Haylton Jorge Suaid ${ }^{2}$, Adauto José Cologna ${ }^{3}$, Antonio Carlos Pereira Martins ${ }^{4}$, Silvio Tucci Jr
} Antonio Antunes Rodrigues ${ }^{5}$, José Anastácio Dias Neto ${ }^{6}$

\begin{abstract}
Suaid HJ, Cologna AJ, Martins ACP, Tucci Jr S, Rodrigues AA, Dias Neto JA. Ação do citrato de sildenafil sobre a função uretral de ratas com desnervação vesical. Acta Cir Bras [serial online] 2003 vol 18 suppl 5. Disponível em www.scielo.br/acb.

RESUMO - O óxido nítrico atua como neurotransmissor não adrenérgico e não colinérgico na bexiga e na uretra. Sua forma de ação se faz pela ativação da guanilatociclase responsável pela transformação de GMP em GMPc que promove o relaxamento da fibra muscular lisa. $\mathrm{O}$ citrato de sildenafil causa aumento do GMPc, através da inibição de fosfodiesterases, que hidrolisam o GMPc. Assim, o objetivo do experimento foi verificar sua ação na uretra. Métodos: Estudou-se 6 ratas com peso aproximado de $200 \mathrm{~g}$. A anestesia foi com uretana na dose de $1,25 \mathrm{mg} / \mathrm{kg}$. As cistometrias, em número de 3, foram realizadas através de cistostomia com cateter P50. A primeira logo após a cistostomia; a segunda depois da desnervação cirúrgica da bexiga e a terceira uma hora após a infusão gástrica do citrato de sildenafil. $\mathrm{O}$ sistema de registro das pressões constou de uma bomba de infusão contínua regulada para $0,3 \mathrm{ml} /$ minuto conectada em Y com o cateter P50 e a um polígrafo Narco-Bioystem. Nas cistometrias avaliou-se as pressões vesicais máxima e mínima nos momentos: normais-(I); desnervadas (II) e desnervadas com citrato de sildenafil na dose de $1 \mathrm{mg} / \mathrm{kg}$ (III). A análise estatística foi feita pelo método de Wilcoxon. Resultados: As médias das pressões máximas (pma) foram: momentos I ( $\mathrm{x}=86,6-\mathrm{SD}=10,1)$; momentos II $(\mathrm{x}=42,6-\mathrm{SD}=15,0)$ e momentos III ( $\mathrm{x}=30,8-\mathrm{SD}=12,4)$. As médias das pressões mínimas (pmi) foram: momentos I $(\mathrm{x}=72,1-\mathrm{SD}=18,9)$; momentos II $(\mathrm{x}=31,1-\mathrm{SD}=9,8)$; momentos III $(\mathrm{x}=14,5-\mathrm{SD}=9,5)$. A análise estatística entre as pma e pmi no mesmo grupo mostrou $\mathrm{p}<0,01$ para o grupo III, sendo maior que 0,05 para os demais. A comparação das pma revelou p< 0,002 entre os momentos I-II e I-III, sendo maior que 0,05 entre os momentos II e III. A comparação das pmi mostrou p< 0,004 entre os momentos I-II; $\mathrm{p}<0,002$ entre os momentos I -III e p <0,01 entre os momentos II-III, havendo portanto nível de significância entre os 3 momentos. Conclusões: 1) A desnervação vesical promove queda nas pressões uretrais máxima e mínima; 2) O Citrato de sildenafil amplia a faixa pressórica de trabalho da uretra devido a diminuição da pressão mínima; 3) O citrato de sildenafil não altera a pressão uretral máxima depois da desnervação.

DESCRITORES: Uretra. Oxido Nítrico. Citrato de Sildenafil.
\end{abstract}

\section{INTRODUÇÃo}

O óxido nítrico está sendo considerado um neurotransmissor não adrenérgico - não colinérgico, da inervação aferente de alguns órgãos e dentre eles encontra-se a bexiga, uretra e penis ${ }^{1,2}$. $\mathrm{Na}$ uretra sua ação tem sido estudada tanto na musculatura lisa quanto na estriada. Assim, encontrou-se que a via NO tem importante papel no relaxamento do esfincter interno durante as contrações vesicais rítmicas ${ }^{3}$. Estudos imunohistoquímicos mostraram maior atividade da NOS e NADPH na uretra proximal, sendo também encontrada a presença de nervos nitrinérgicos e NOS no sarcolema de fibras estriadas do esfincter externo ${ }^{4}$. A via de atuação do NO se faz pela ativação da guanilase ciclase que transforma o GMP em GMPc, que atua como miorrelaxante. A hidrolise do GMPc é feita por uma série de enzimas chamadas fosfodiesterases. O citrato de Sildenafil é um bloqueador da fosfodiesterase 5, que promove o aumento do teor de $\mathrm{GMPc}^{5}$. Na uretra pré-contraída de camundongos provoca aumento do tempo de relaxamento e da amplitude do estímulo elétrico ${ }^{6}$ "in vitro".

\section{OBJETIVO}

Em vista aos achados acima descritos, resolveuse verificar a ação do citrato de sildenafil sobre a função uretral de ratas.

\section{MÉTODOS}

1) ANIMAIS

Usou-se 6 ratas da raça Wistar, com peso aproximado de 200 gramas.

\section{2) ANESTESIA}

As ratas foram anestesiadas com uretana na dosagem de 1,250 $\mathrm{mg}$ por kilo de peso, via intraperitonial.

\section{3) PROCEDIMENTOS}

a) Cistostomia: Inicialmente foi feita tricotomia abdominal. Depois a laparotomia com exposição,

1. Trabalho realizado no setor de Cirurgia Experimental do Departamento de Cirurgia e Anatomia - HCFMRP-USP

2. Prof. Associado do Depto de Cirurgia e Anatomia - Disciplina de Urologia da FMRP-USP

3. Prof. Doutor do Depto de Cirurgia e Anatomia - Disciplina de Urologia da FMRP-USP

4. Prof. Titular do Depto de Cirurgia e anatomia - Disciplina de Urologia da FMRP-USP

5. Pós-Graduando do Depto de cirurgia e anatomia da FMRP - USP

6. Médico Residente da Disc. De Urologia do Depto de Cirurgia e Anatomia da FMRP-USP 